\title{
Tratamento de cisto congênito em anexos oculares
}

\author{
[Treatment of cystic disease in ocular adnexa]
}

\section{"Relato de Caso/Case Report"}

\author{
Mariana Araújo Rocha ${ }^{1 *}$, Jussara Sampaio Quintela ${ }^{1}$, Keytyanne de Oliveira Sampaio ${ }^{2}$, \\ Roberio Gomes Olinda ${ }^{3}$, Reginaldo Pereira de Sousa Filho ${ }^{3}$
}

\begin{abstract}
${ }^{1}$ Faculdade de Veterinária (FAVET), Universidade Estadual do Ceará (UECE), Fortaleza-CE, Brasil.
${ }^{2}$ Departamento de Medicina Veterinária, Universidade Federal Rural de Pernambuco (UFRPE), Recife-PE, Brasil.

${ }^{3}$ Centro de Ciências de Saúde - Medicina Veterinária, Universidade de Fortaleza - UNIFOR, Fortaleza-CE, Brasil.

${ }^{4}$ Médico Veterinário Autônomo, Catus Medicina Felina, Fortaleza-CE, Brasil.

*Autor para correspondência/Corresponding author: E-mail: mariana4981@ hotmail.com
\end{abstract}

\section{Resumo}

A formação de cisto de glândula lacrimal é uma comorbidade rara em animais domésticos. O objetivo deste relato é descrever um caso de um cão com cisto de glândula lacrimal. Um cão, macho, sem raça definida, com onze meses de idade, foi atendido com histórico de formação vesicular subpalpebral direita com evolução de três meses. Realizou-se exames complementares como citologia do conteúdo da vesícula e radiografia do crânio do animal. De acordo com a localização topográfica, idade e histórico do animal, suspeitou-se de cisto de glândula lacrimal, sendo encaminhado para exérese cirúrgica e realização de exame histopatológico da peça cirúrgica, onde se confirmou o diagnóstico de cisto de glândula lacrimal. A ressecção cirúrgica da glândula e do cisto lacrimal mostrou-se ser uma medida terapêutica curativa neste caso, sem haver comprometimento na secreção lacrimal.

Palavras-chave: anexos oculares; canino; histopatologia.

\begin{abstract}
Cysts of the lacrimal gland are rare in domestic animals. Hence, the objective of this report is to describe a case of a dog with lacrimal gland cyst. A dog, male, mixed-breed, at eleven months of age, presented with a history of right subpalpebral vesicular formation, which developed in three months. Complementary tests were performed, such as cytology of the vesicle content and x-ray of the skull. According to the topographic location, age and anamneses, a lacrimal gland cyst was suspected. Then, surgical excision and histopathological examination of the surgical specimen were performed, where the diagnosis of lacrimal gland cyst was confirmed. Surgical resection of the gland and lacrimal cyst was an adequate curative therapeutic measure in this case without compromising lacrimal secretion.
\end{abstract}

Keywords: ocular attachments; canine; histopathology.

\section{Introdução}

O sistema lacrimal composto por glândulas serosas, seromucosas e ductos, é responsável pela drenagem de secreções a partir do saco conjuntival, além da produção do filme lacrimal, que consiste de três camadas diferentes, a superficial externa composta de lipídios, a intermediária composta por componentes aquosos e a interna composta de mucina (Dartt, 2004).

As principais glândulas responsáveis pela produção das lágrimas são a glândula principal (orbital) e a glândula acessória (nictitante), localizadas na região periorbital na face dorsolateral da órbita e na terceira pálpebra, 
respectivamente, sendo estas constituídas de estruturas tubuloacinares com unidades secretoras, ductos e tecido conjuntivo (Cabral et al., 2005; Samuelson, 2013).

A formação de cistos envolvendo o tecido lacrimal podem se originar da órbita ou da membrana nictitante, sendo raro em animais domésticos. A literatura relata a predominância dessa comorbidade em cães jovens, com até um ano de idade (Ota et al., 2009; Delgado, 2012).

O cisto lacrimal pode causar uma distensão da conjuntiva e protrusão para a fenda palpebral ou pode se expandir dentro da órbita e deslocar o globo ocular, de acordo com o seu local de origem. As possíveis causas das formações císticas incluem traumas, corpo estranho, processo inflamatório nos ductos, neoplasias ou anomalias de desenvolvimento (Giuliano, 2013).

Através da topografia e da apresentação clínica característica de cisto da glândula lacrimal, o diagnostico presuntivo é clínico, sendo confirmado por histopatologia e/ou imunohistoquímica (Betharia et al., 2002; Ota et al., 2009). A excisão cirúrgica tem sido eficaz e curativa como tratamento em cães com esta estrutura cística (Ota et al., 2009; Delgado, 2012). Assim, o objetivo deste trabalho é relatar o tratamento de um caso de cisto de glândula lacrimal em um cão.

\section{Descrição do caso}

Foi atendido no Hospital veterinário um cão, macho, sem raça definida (SRD), 11 meses de idade, com histórico de aumento de volume na região superior da orbita ocular direita com evolução gradativa há três meses. No exame clínico foi constatada uma formação vesicular subpalpebral superior direita, acometendo a conjuntiva palpebral e ocular (Figura 1), sem sinal de dor, e o paciente encontrava-se normohidratado, normocorado, escore corporal 3 (escala de 1-5), temperatura corporal normal, palpação abdominal sem alterações $\mathrm{O}$ conteúdo da vesícula foi puncionado e coletou-se cerca de $20 \mathrm{ml}$ de líquido com a coloração amarelo-acastanhado (Figura 2).

O material foi encaminhado para a citologia, com resultado característico de transudado modificado, sem maiores achados conclusivos. Solicitou-se uma radiografia crânio latero-lateral direita e rostro-caudal, não sendo identificada nenhuma alteração em estrutura óssea. Com base na localização topográfica da estrutura, na idade do animal e na ausência de alterações sistêmicas, suspeitou-se de cisto da glândula lacrimal principal de etiologia congênita, sendo o paciente encaminhado para exérese da formação cística e da glândula acometida. No pré-cirúrgico, foram realizados hemograma completo, dosagem sérica de creatinina, alanina aminotransferase (ALT) e albumina, com resultados sem alterações.

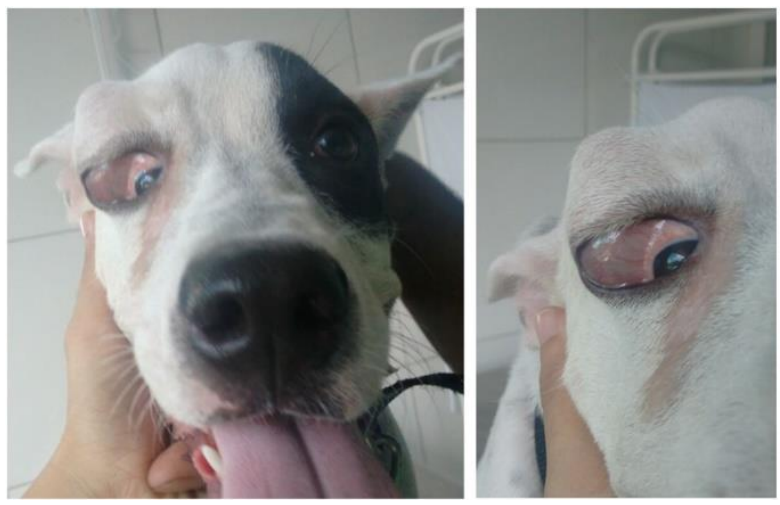

Figura 1. Olho direito do cão com presença de inchaço na região subpalpebral.

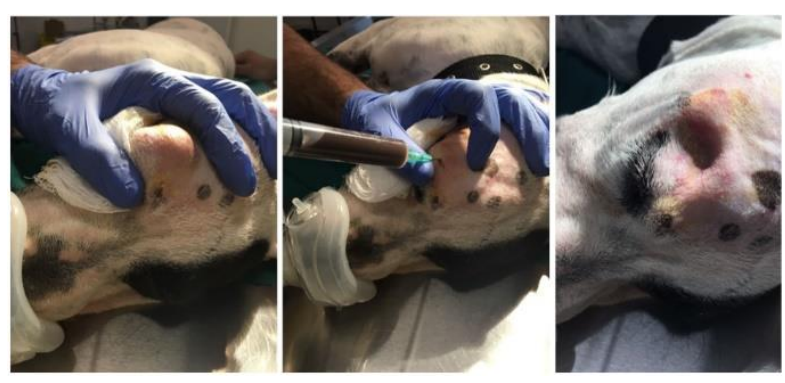

Figura 2. Conteúdo líquido com coloração amareloacastanhado, obtido na punção da vesícula subpalpebral do cão.

No procedimento cirúrgico, iniciou-se a dissecção da estrutura, através de uma incisão subpalpebral, seccionando e separando o cisto de forma encapsulada do tecido conjuntival. $\mathrm{O}$ ducto da glândula lacrimal principal foi transfixado e obliterado com o uso de fio Polidiaxonona-0. Após a remoção da glândula, foi realizada abolição do espaço morto com sutura simples de padrão continuo com fio Polidiaxonona 3-0, e realizado sutura da pele em padrão continuo intradérmico, utilizando o mesmo fio. Para o pós-cirúrgico foi prescrito meloxican $(0,1 \mathrm{mg} / \mathrm{kg})$ por três dias e tramadol $(2 \mathrm{mg} / \mathrm{kg})$ por cinco dias e colírio a base de ciprofloxacino a 1\% (1 gota quatro vezes ao dia), por 14 dias.

O animal retornou após sete dias, onde foi observado a presença de seroma, sendo indicado dar continuidade ao tratamento com meloxican por mais dois dias. Após 20 dias o paciente retornou para reavaliação cirúrgica e retirada dos pontos, onde já não foi mais observado seroma na região. 
Três meses do procedimento, o cão retornou apresentando regressão total do quadro.

O material excisado foi encaminhado para exame histopatológico, onde se observou diversas secções de tecido compatível com tecido conjuntival, em algumas seç̧ões com representação de tecido glandular, além de um processo inflamatório sem presença de agentes infecciosos. Em substância conjuntival superficial observou-se moderada inflamação perivascular a intersticial. Em alguns campos observou-se discreta a moderada distensão de ductos de glândulas (Figura 3).

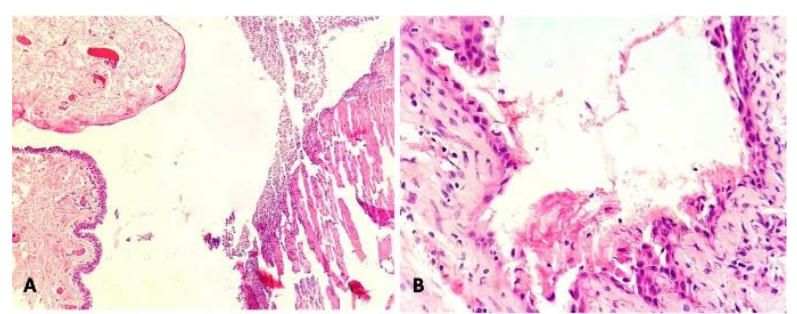

Figura 3. Aspecto histológico de cisto ductal em glândula lacrimal. A- Evidenciam-se espaços císticos delimitados por epitélio colunar e no seu interior há material necrótico amorfo, eosinofílico e infiltrado neutrofílico. B - Há marcada dilatação cística e descamação das células epiteliais.

Foi ainda realizado colorações especiais de ácido periódico de Schiff (PAS) e Grocott-Gomori, onde não foi observado indícios de agentes infecciosos. Em meio ao material fibrinoide observou-se -se áreas PAS-positivas, podendo corresponder a material mucoide. Concluiu-se que a área focal de marcante exsudação fibrinosupurativa em meio à substância PASpositiva, juntamente com o histórico clínico, sugere-se tratar de parede de lesão de cisto de glândula lacrimal.

\section{Discussão}

A presença de massas na região orbital e no canto medial do olho incluem como diagnósticos diferenciais o cisto de origem de glândula lacrimal (Delgado, 2012), cistos do tecido anexial orbital (conjuntiva, ducto nasolacrimal, canalículo e saco lacrimal) (Davidson e Blanchard, 1991; Giuliano, 2013), abscesso (Homma e Schoster, 2000), dacriocistite (Voelter-Ratson et al., 2015) e neoplasias (Ralic et al., 2014). No presente relato foi diagnosticado um caso de cisto da glândula lacrimal principal em um cão.

A patogênese da estrutura cística em caninos ainda não está bem elucidada, porém, estudos em humanos sugerem que a origem seja decorrente de quadros inflamatórios ou trauma na região periorbital, resultando em uma hipersecreção e destruição da contratilidade neuromuscular do ducto da glândula lacrimal, enfraquecendo as paredes do ducto e ocasionando uma dilatação passiva do ducto com a formação do cisto (Betharia et al., 2002). Neste relato não foi observado histórico de trauma aparente ou processo infeccioso inicial.

A glândula lacrimal e seus ductos se desenvolvem a partir de um botão isolado do ectoderma de superfície e a formação de cistos congênitos pode ser decorrente de uma má formação embrionária (Wilcock e Njaa, 2015). Esses cistos, quando de etiologia congênita, são caracterizados por um aumento progressivo, acompanhando o crescimento do globo ocular de animais jovens (Ota et al., 2009; Delgado, 2012). No presente relato, a localização do cisto, a idade do paciente e a ausência de histórico de trauma corroboram com a literatura citada.

As formações císticas nas glândulas lacrimais são relatadas em cães da raça Mastim Napolitano, Labrador Retriever e Pastor Alemão, com faixa etária de três meses a um ano (Cullen e Grahn, 2003; Ota et al., 2009; Delgado, 2012), nesse estudo foi observado essa formação em um canino de 11 meses de idade e sem raça definida.

Assim como observado, as anormalidades ósseas são achados incomuns, visto que essa enfermidade não acomete o tecido ósseo, não sendo portanto observada alterações por meio de radiografias (Cullen e Grahn, 2003). A injeção de contraste na região é indicada para delimitar o tamanho do cisto e possibilita a visualização de possíveis comunicações com outros tecidos, além de também observar se há presença de corpo estranho (Ota et al., 2009).

A exérese completa da glândula lacrimal principal, assim como relatado por Ota et al. (2009) e Delgado (2012), demonstrou ser um tratamento curativo, já que o paciente não apresentou nenhuma reicidiva após três meses do procedimento cirúrgico. A aplicação de tetraciclina intralesional é utilizada para o tratamento de cistos perioculares envolvendo a glândula salivar zigomática, devido a sua ação fibrosante, porém, em consequência de seu potencial necrotizante, não é recomendada como tratamento para animais com cistos de glândulas lacrimais (Giuliano, 2013).

A glândula lacrimal orbital é a maior e apresenta um parênquima secretor superior ao da glândula nictitante em cães (Cabral et al., 2005). A 
excisão parcial da glândula principal pode ocasionar uma ceratoconjuntivite seca, devido ao comprometimento da secreção lacrimal (Delgado, 2012), entretanto, Busnardo et al. (2010) relatou que houve compensação da produção aquosa do filme lacrimal a partir do restante da glândula que não foi removida. Em um estudo sobre a remoção unilateral da glândula orbital em macacos-esquilos, mostrou que o filme lacrimal da glândula acessória foi suficiente para manter uma camada estável de secreções na córnea (Maitchouk et al., 2000), corroborando com o presente relato, visto que neste não houve a presença de complicações póscirúrgicas no animal através do exame clínico, sugerindo-se que a glândula acessória e outros componentes do sistema lacrimal, podem suprir a produção lacrimal.

A caracterização do cisto lacrimal na histopatologia corresponde principalmente pela presença de inflamação da parede revestida por epitélio cuboide achatado do cisto, e pela dilatação de ácinos e ductos da glândula lacrimal, devido à estase secretora (Giuliano, 2013). No caso presente, foram visualizadas numerosas células inflamatórias e distensão de ductos de glândulas, sendo compatível com o cisto de glândula lacrimal. A presença de epitélio cuboide na parede do cisto e atrofia de ácinos glandulares foi observada em um estudo (Delgado, 2012).

Assim como relatado por Ota et al. (2009), a imunohistoquímica confirma a presença do cisto de glândula lacrimal, através da coloração positiva para actina do músculo liso, confirmando a presença de células mioepiteliais no revestimento do cisto, sendo, essa uma característica importante para o diagnóstico dessa afecção. O diagnóstico foi realizado a partir do exame histopatológico associado a localização da estrutura e a idade jovem do animal.

Casos de cisto na glândula lacrimal são raros, sendo um diagnóstico diferencial para cães jovens que apresentam inchaço localizado na região periorbital na face dorsolateral da órbita. $\mathrm{O}$ histórico e o exame clínico acompanhado da histopatologia e imuno-histoquímica são as principais ferramentas de diagnóstico. A excisão cirúrgica tem sido o tratamento mais eficaz e curativo, entretanto, deve-se averiguar a presença de ceratoconjuntivite seca ou de outras comorbidades como complicações pós-cirúrgicas.

\section{Conclusão}

O exame clínico associado ao histopatológico de cisto da glândula lacrimal é fundamental para elucidar o diagnóstico de animais com essa comorbidade. A excisão cirúrgica mostrou-se eficaz e curativa para a resolução do cisto lacrimal, não sendo detectadas complicações como ceratoconjuntivite seca.

\section{Conflito de Interesse}

Os autores declaram não existir conflito de interesse.

\section{Referências}

Betharia, S.M; Pushker, N.; Sharma, V.; Sen, S. Simple dacryops: a case series and review of the literature. Ophthalmologica, 216(5): 372376. 2002.

Busnardo, C.A.; Eurides, D.; Baugarten, L.B.; Silva, L.A.F.; Beletti, M.E.; Guimaraes, E.D.; Alves, L.B.; Oliveira, B.J.N.A.; Souza, L.A.; Nadri, A.B.; Gonçalves, G.F. Produção de lágrima após exérese parcial da glândula lacrimal principal em cães. Acta Scientiae Veterinariae, 38(2): 171-176, 2010.

Cabral, V.P.; Laus, J.L.; Dagli, M.L.Z.; Pereira, G.T.; Talieri, I.V.; Monteiro, E.R.; Mamede, F.V. Canine lacrimal and third eyelid superficial glands' macroscopic and morphometric characteristics. Ciência Rural, 35(2): 391-397, 2005.

Cullen, C.L.; Grahn, B.H. Diagnostic ophthalmology. Congenital medial canthal cyst. The Canadian Veterinary Journal, 44(11): 935-937, 2003.

Dartt, D.A. Control of mucin production by ocular surface epithelial cells. Experimental Eye Research, 78(2): 173-185, 2004.

Davidson, H.J.; Blanchard, G.L. Periorbital epidermoid cyst in the medial canthus of three dogs. Journal of the American Veterinary Medical Association, 198(2): 271-272, 1991.

Delgado, E. Dacryops of the lacrimal gland in a dog. Veterinary Ophthalmology, 16(2): 153-158, 2012.

Giuliano, E.A. Diseases and surgery of the canine lacrimal secretory system. In: Gelatt, K.N.; Gilger, B.C.; Kern, T.J. Veterinary ophthalmology. $5^{\text {th }}$ ed. United Kingdom: John Wiley \& Sons, 2013. p. 912-944.

Homma, K.; Schoster, J.V. Anaerobic orbital abscess/cellulitis in a Yorkshire Terrier dog. The Journal of Veterinary Medical Science, 62(10): 1105-1107, 2000. 
Maitchouk, D.Y.; Beuerman, R.W.; Ohta, T.; Stern, M.; Varnell, R.J. Tear production after unilateral removal of the main lacrimal gland in Squirrel Monkeys. Archives of Ophthalmology, 118(2): 246-252, 2000.

Ota, J.; Pearce, J.W.; Finn, M.J.; Johson, G.C.; Giuliano, E.A. Dacryops (lacrimal cyst) in three young Labrador Retrievers. Journal of the American Animal Hospital Association, 45(4): 191-196, 2009.

Ralic, M.; Vasic, J.; Jovanovic, M.; Cameron, B. Retrobulbar chondrosarcoma in dog. Open Veterinary Journal, 4(1): 51-55, 2014.
Samuelson, D.A. Ophtalmic anatomy. In: Gelatt, K.N.; Gilger, B.C.; Kern, T.J. Veterinary ophthalmology. 5a ed. United Kingdom: John Wiley \& Sons, 2013. p. 39-170.

Voelter-Ratson, K.; Hagen, R.; Grundmann, S.; Spiess, B.M. Dacryocystitis following a nasolacrimal duct obstruction caused by an ectopic intranasal tooth in a dog. Veterinary ophthalmology, 18(5): 433-436, 2015.

Wilcock, B.P.; Njaa, B.L. Special senses. In: Maxie, M.G. Jubb, Kennedy and Palmer's pathology of domestic animals. $6^{\text {th }}$ ed., vol 1. United Kingdom: Saunders, 2015. p. 408-488. 